Supporting Information for

\title{
Kinetically-Controlled Synthesis of Rhodium Nanocrystals with Different Shapes and A Comparison Study of Their Thermal and Catalytic
}

\section{Properties}

Ming Zhao ${ }^{\$}{ }^{\$}$ Zitao Chen, ${ }^{\$ \$}$ Yifeng Shi, ${ }^{\S}$ Zachary D. Hood $,{ }^{\dagger, \# ~ Z h i h e n g ~ L y u ~},{ }^{\dagger}$ Minghao Xie, ${ }^{\dagger}$ Miaofang Chi, ${ }^{\#}$ Younan $\mathrm{Xia}^{*}, \dagger, \xi, \$$

†School of Chemistry and Biochemistry, Georgia Institute of Technology, Atlanta, Georgia 30332, United States

\$The Wallace H. Coulter Department of Biomedical Engineering, Georgia Institute of Technology and Emory University, Atlanta, Georgia 30332, United States

${ }^{\S}$ School of Chemical and Biomolecular Engineering, Georgia Institute of Technology, Atlanta, Georgia 30332, United States

${ }^{\#}$ Center for Nanophase Materials Sciences, Oak Ridge National Laboratory, Oak Ridge, Tennessee 37831, United States

${ }^{\$}$ These authors contributed equally to this work.

"Corresponding author: younan.xia@bme.gatech.edu 


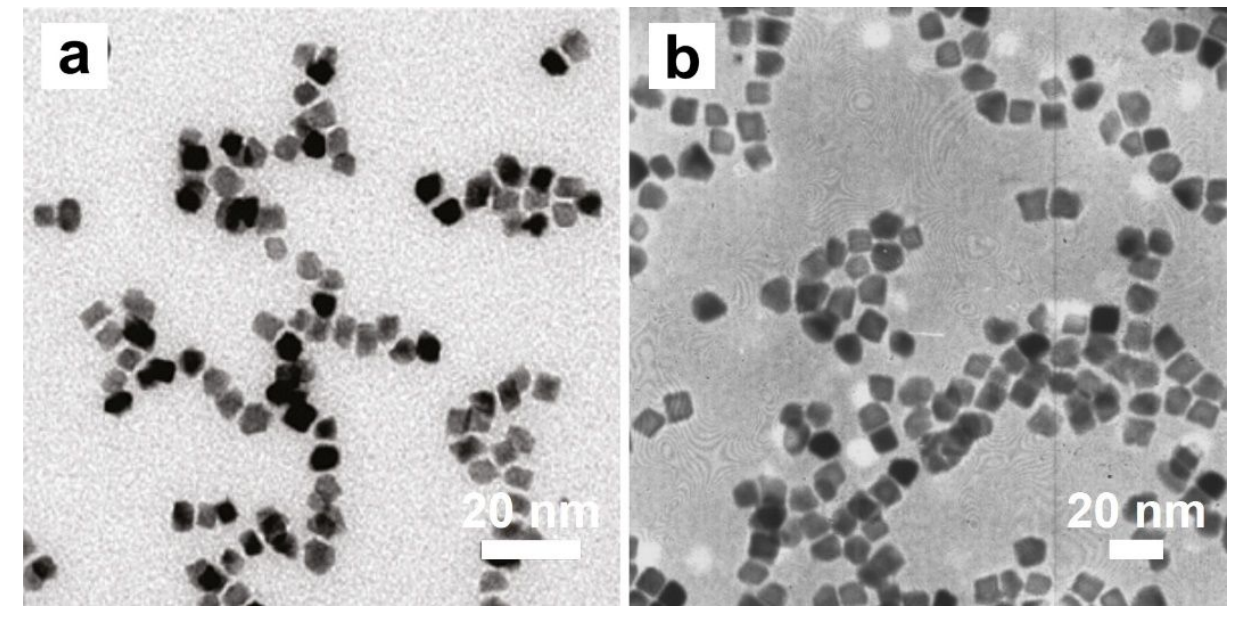

Figure S1. TEM images of the typical Rh octahedral nanocrystals reported in literature. ${ }^{1,2}$ The syntheses involved the reduction of $\mathrm{RhCl}_{3}$ by (a) poly(ethylene glycol) (M.W. = 300) at a reflux temperature of $325{ }^{\circ} \mathrm{C}$ and (b) $\mathrm{EG}$ in the presence of $\mathrm{AgNO}_{3}(\mathrm{Ag} / \mathrm{Rh}=0.011 \%)$ at $160{ }^{\circ} \mathrm{C}$, respectively. It should be pointed out that the nanocrystals shown in panel (b) were inevitably contaminated by Ag atoms, corresponding to an alloy rather than pure Rh. (a) Reprinted with permission from ref 1. Copyright 2011 American Chemical Society. (b) Reprinted with permission from ref 2. Copyright 2011 Elsevier. 


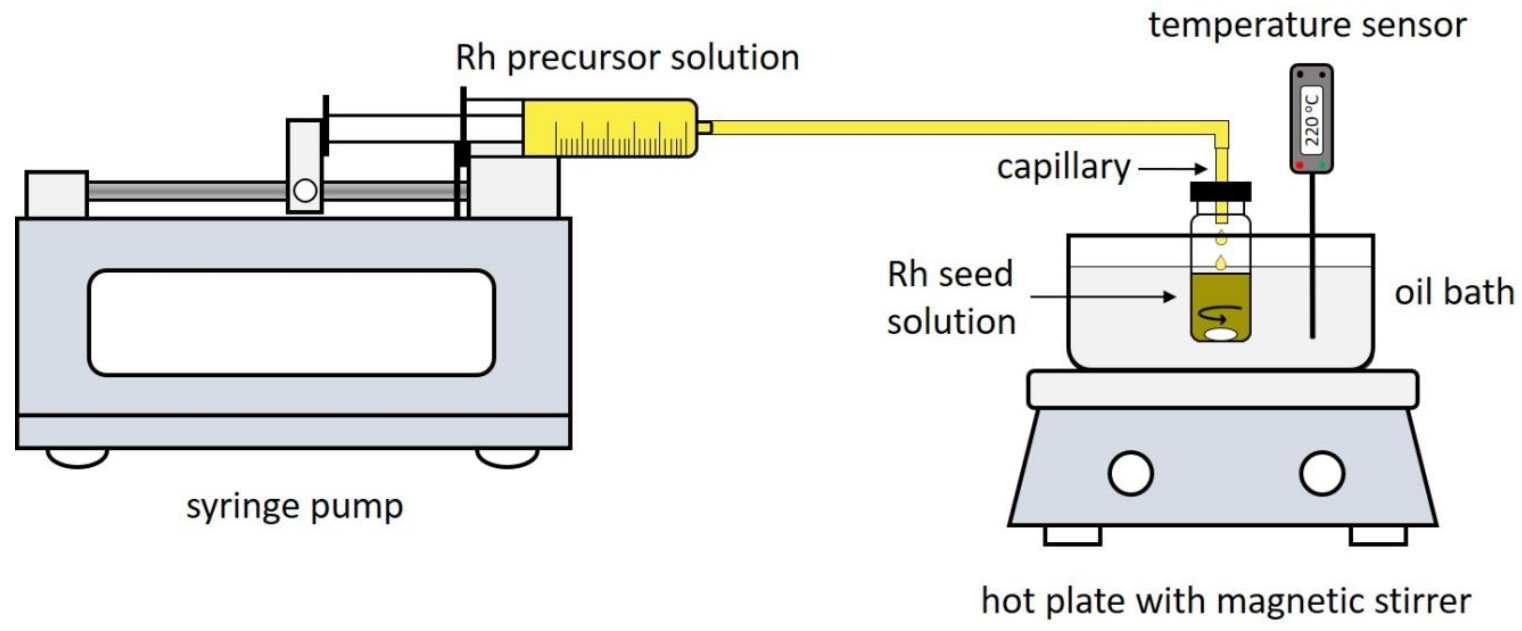

Figure S2. Schematic illustration showing the setup used for the synthesis. Regarding the scaleup synthesis, the $20-\mathrm{mL}$ vial was replaced by a $100-\mathrm{mL}$ flask, while the precursor solution was still added dropwise. 

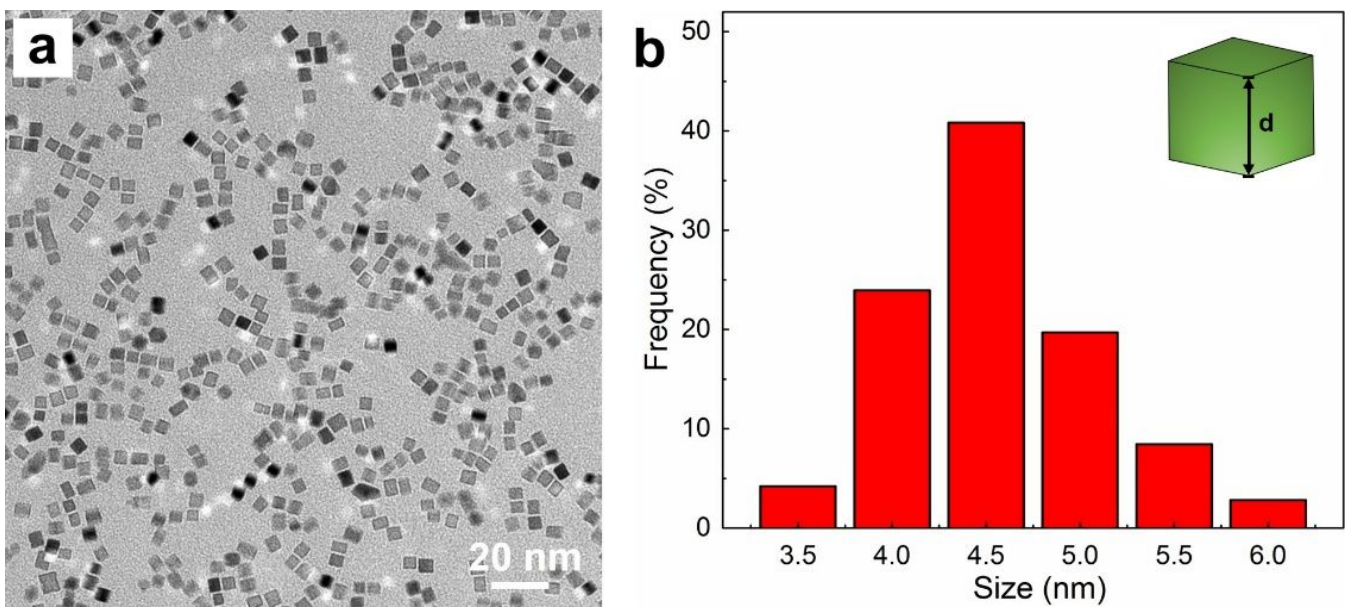

Figure S3. (a) TEM image of the Rh nanocubes used as seeds for the synthesis of octahedra. (b) Size distribution of the Rh cubic seeds, showing an average edge length of $4.5 \pm 0.4 \mathrm{~nm}$. The inset in panel (b) shows the schematic of a cube and the definition of size. 


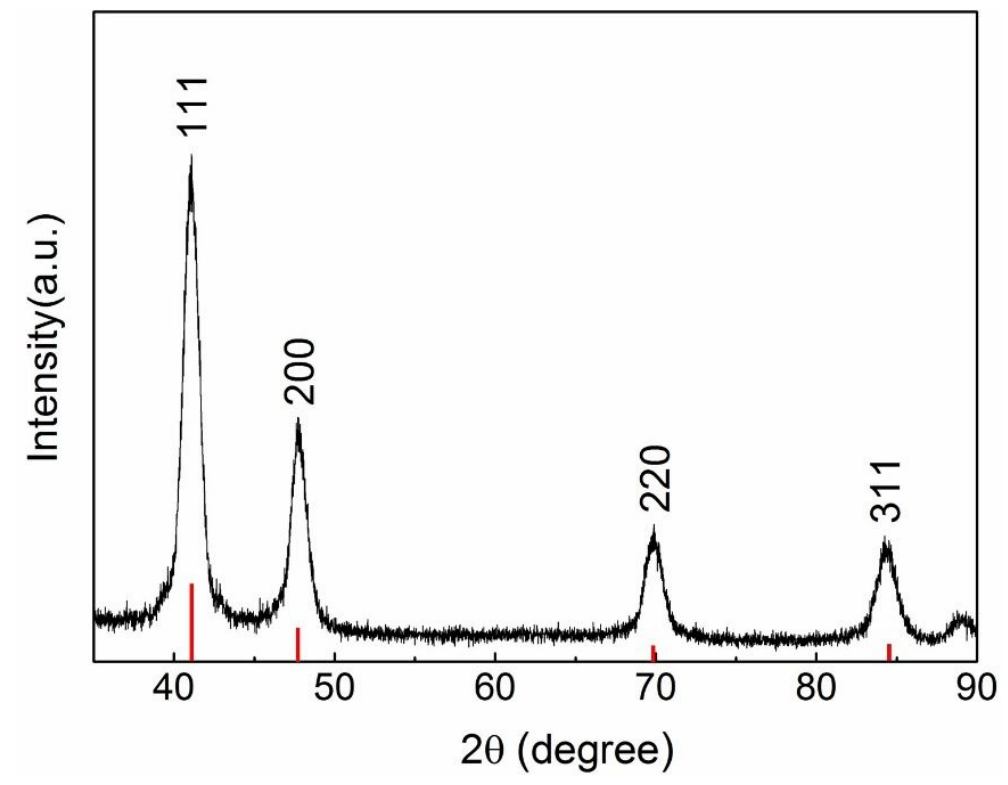

Figure S4. XRD pattern of the Rh octahedra prepared using the standard protocol, showing the formation of an $f c c$ phase. The characteristic peaks of $f c c \mathrm{Rh}$ are indicated by red bars at the bottom. 


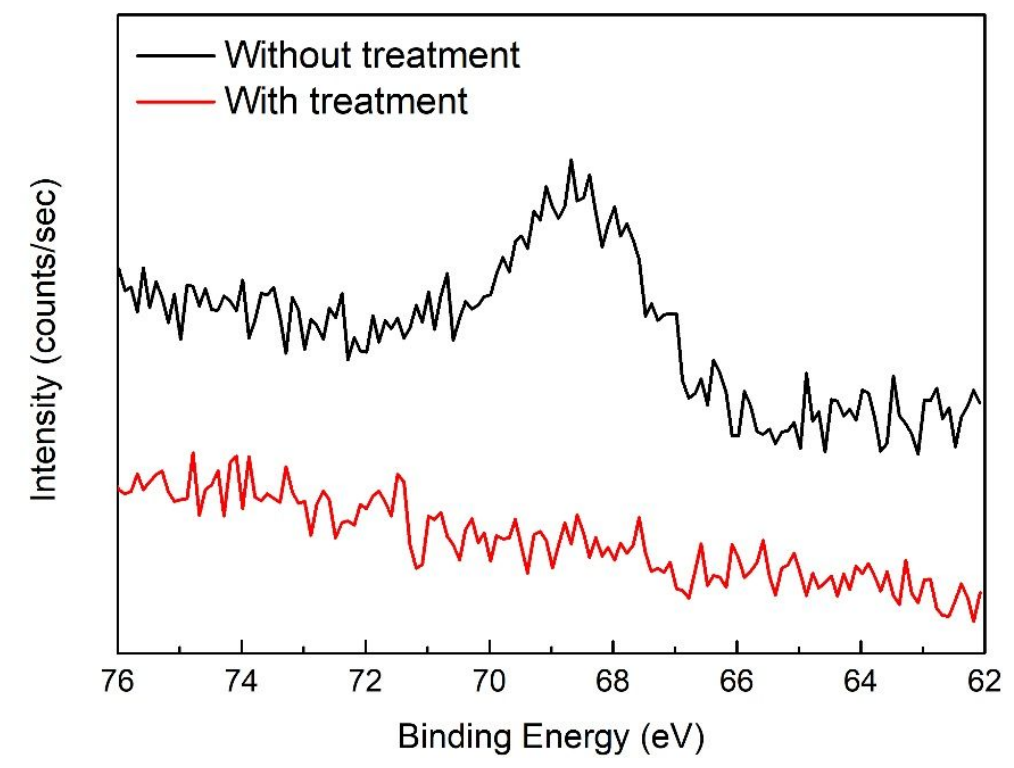

Figure S5. Br $3 \mathrm{~d}$ XPS spectra taken from the 4.5-nm $\mathrm{Rh}$ cubic seeds with and without, respectively, going through the treatment at $220^{\circ} \mathrm{C}$ for $2 \mathrm{~h}$. 


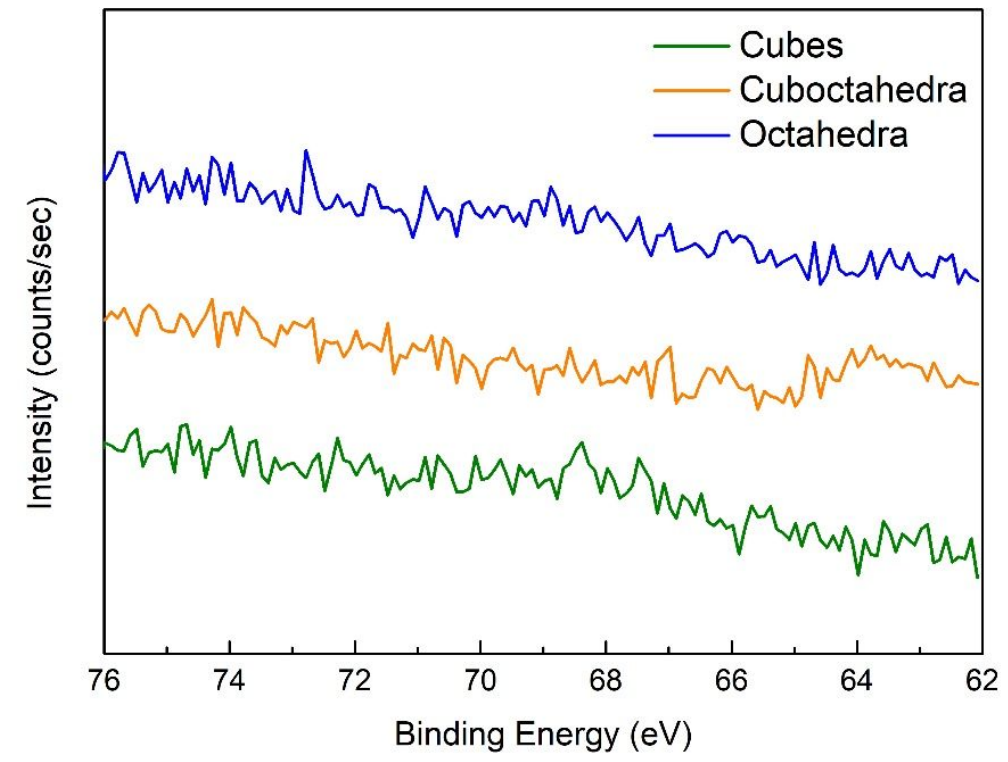

Figure S6. Br 3d XPS spectra taken from the cubes (Figures 1d), cuboctahedra (Figures 2c), and octahedra (Figures 2d) grown from the 4.5-nm Rh cubic seeds. 


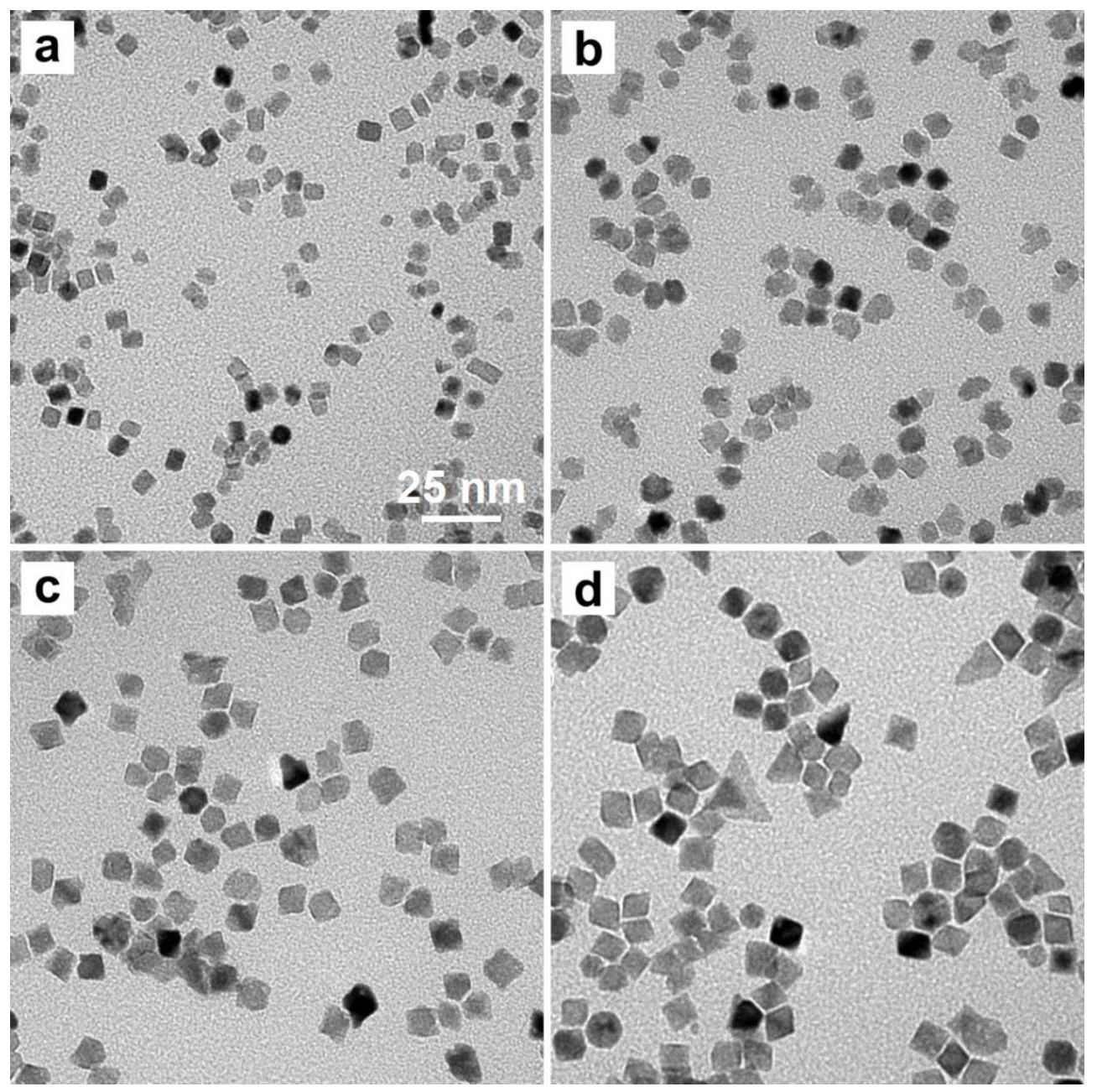

Figure S7. TEM images of the Rh nanocrystals prepared using the standard protocol except for the involvement of different reaction temperatures: (a) 160, (b) 180, (c) 200, and (d) $240^{\circ} \mathrm{C}$. 


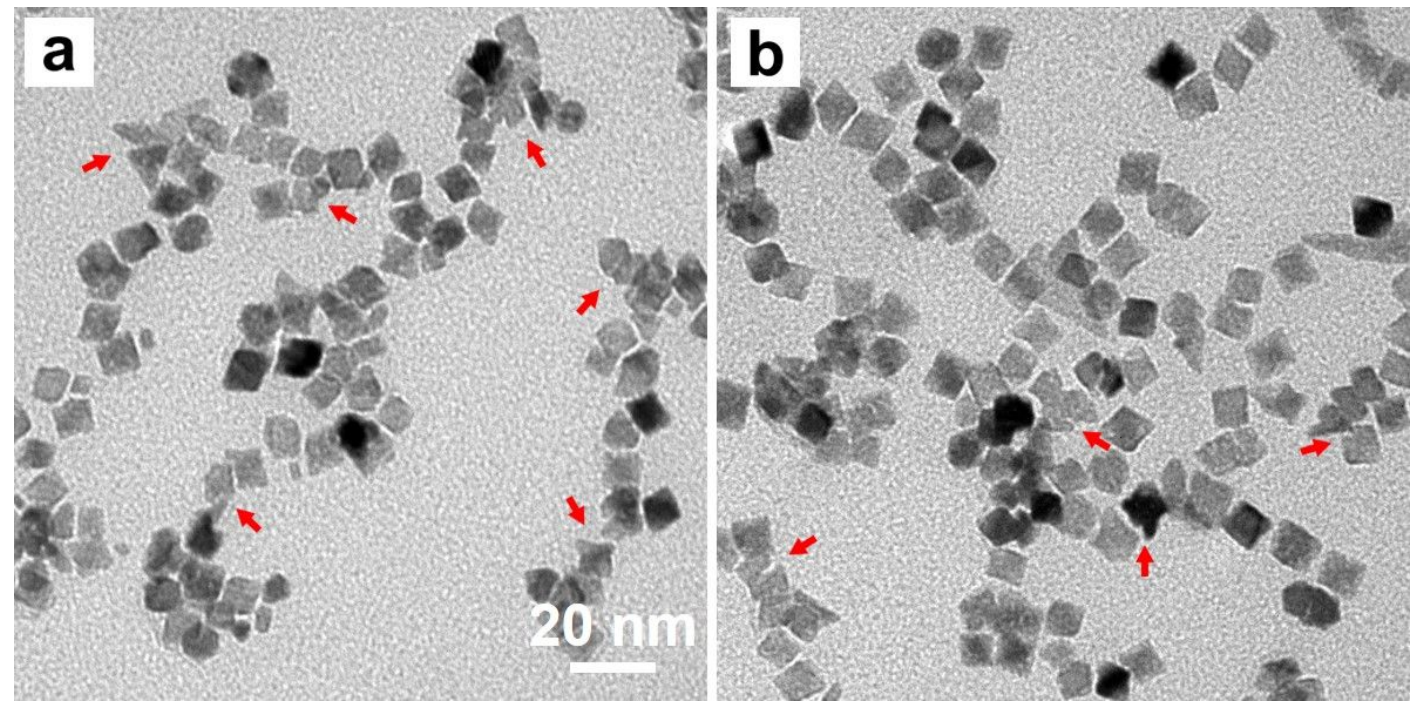

Figure S8. TEM images of the Rh nanocrystals prepared using the standard protocol except for the employment of (a) $\mathrm{Na}_{3} \mathrm{RhCl}_{6}$ and (b) $\mathrm{RhCl}_{3}$, respectively, as the precursor. The red arrows in panels (a) and (b) indicate the typical morphology of Rh nanocrystals when oxidative etching was involved in the synthesis. 


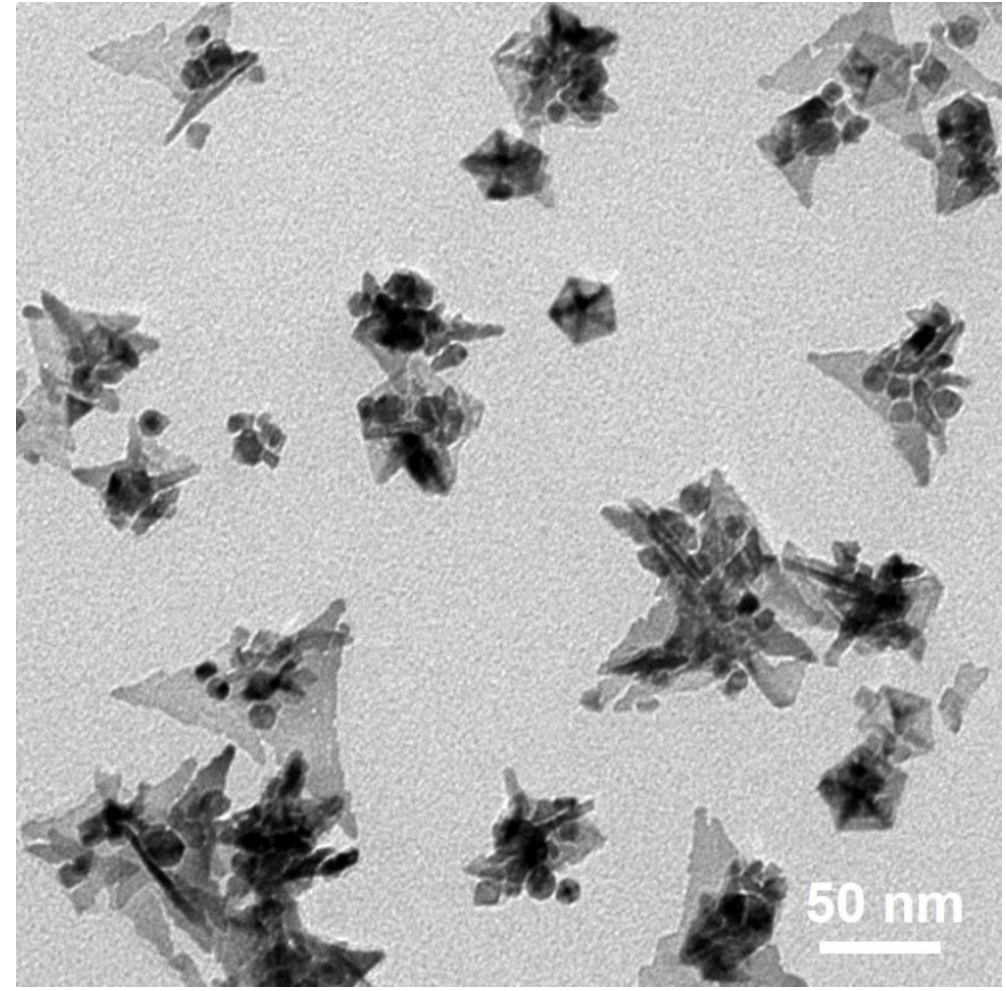

Figure S9. TEM image of the Rh nanocrystals prepared using the standard protocol except for the absence of Rh cubic seeds. 


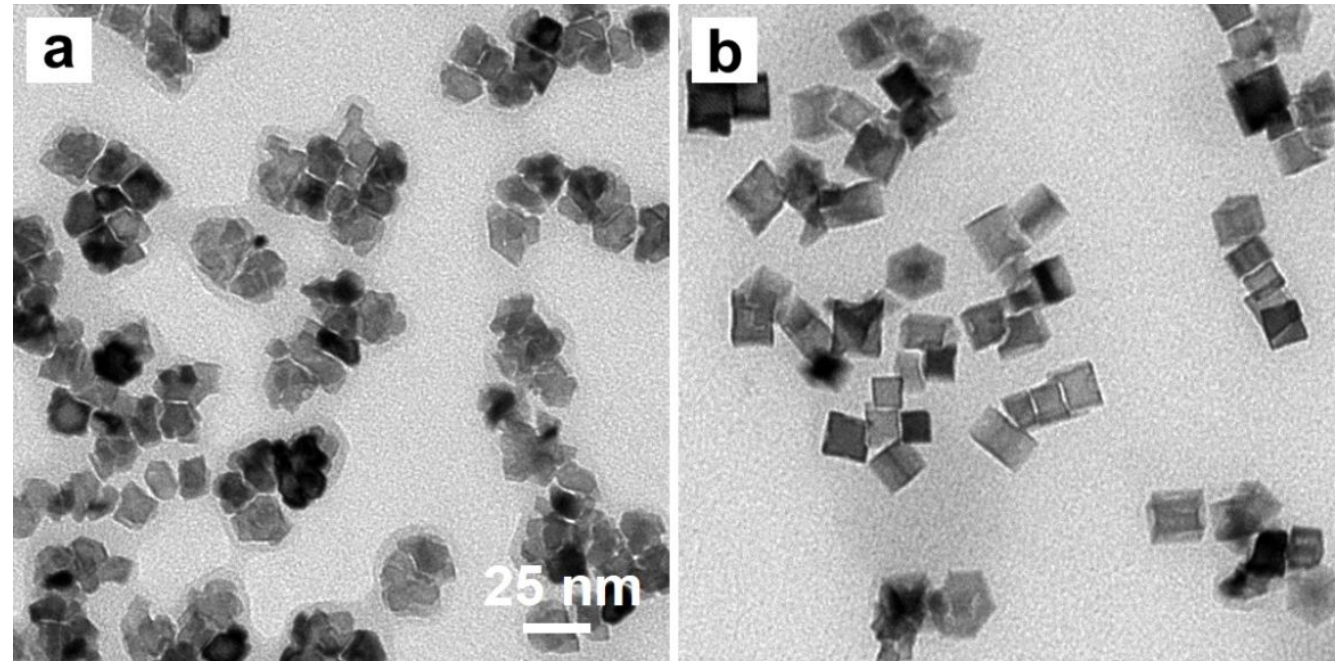

Figure S10. TEM images of the Rh nanocrystals prepared using the standard protocol except for the involvement of 9-nm Rh cubes (Figure 2d) as seeds and at different injection rates for the $\mathrm{Rh}(\mathrm{acac})_{3}$ solution: (a) 5 and (b) $0.5 \mathrm{~mL} \mathrm{~h}^{-1}$, respectively. 

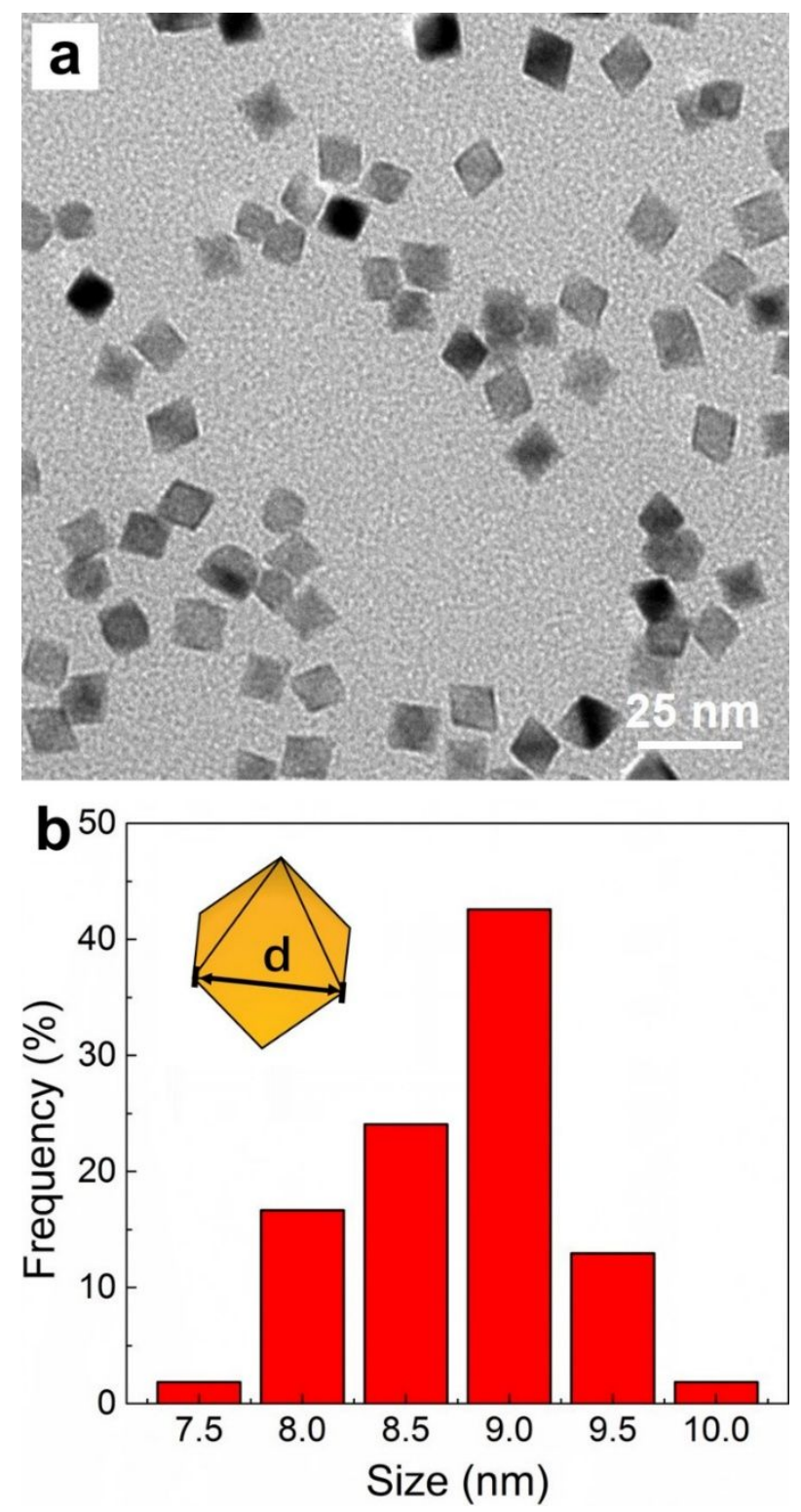

Figure S11. (a) TEM image of the Rh octahedra prepared using the scale-up protocol. (b) Size distribution of the as-synthesized Rh octahedra. The inset in panel (b) shows a schematic of the octahedron and the definition of size. 


\section{REFERENCES}

(1) Biacchi, A. J.; Schaak, R. E. The Solvent Matters: Kinetic versus Thermodynamic Shape Control in the Polyol Synthesis of Rhodium Nanoparticles. ACS Nano 2011, 5, 8089-8099.

(2) Long, N. V.; Chien, N. D.; Hirata, H.; Matsubara, T.; Ohtaki, M.; Nogami, M. Highly monodisperse cubic and octahedral rhodium nanocrystals: Their evolutions from sharp polyhedrons into branched nanostructures and surface-enhanced Raman scattering. J. Cryst. Growth 2011, 320, 78-89. 